\title{
Auroral substorm dynamics and field line resonances
}

\author{
James A. Wanliss ${ }^{1}$ and Robert Rankin ${ }^{2}$ \\ ${ }^{1}$ Embry-Riddle Aeronautical University, Physical Sciences Department, Daytona Beach Fl., 32114, U.S.A. \\ ${ }^{2}$ University of Alberta, Department of Physics, Edmonton, AB, T6G 2J1, Canada
}

(Received April 24, 2002; Revised August 24, 2002; Accepted August 27, 2002)

\begin{abstract}
Magnetospheric substorms frequently have oscillating auroral phenomena associated with them, and which may be related to field line resonances. In this paper we present an analysis of photometer data which displays such oscillations in luminosity. We use data from the Gillam station in the CANOPUS array for April 1,2000. Clear pulsations in magnetometer and photometer data are observed at $2.2 \mathrm{mHz}$. The latitudinal distribution of the photometer pulsations indicates that the auroral luminosity variations, caused by precipitating electrons and protons, are modulated by large scale global compressional modes (field line resonances). The proton and electron auroras were found to oscillate essentially out of phase. As well, the variation of phase across the peak in the luminosity resonance follows the pattern expected for the coupling of resonant Alfvén modes by normal compressional modes in the magnetotail.
\end{abstract}

\section{Introduction}

Oscillating auroral phenomenon have been observed via a wide variety of instruments. Examples include magnetometers (Jacobs et al., 1964), coherent scatter radar (McDiarmid et al., 1994; Fenrich et al., 1995), photometers and all sky imager observations (Samson et al., 1992; Liu et al., 1995; Wanliss et al., 2002; Milan et al., 2001; Lyatsky et al., 1999). In a cursory survey of ten years of photometer data from the Gillam photometer of the CANOPUS array, we found that luminosity oscillations were frequently associated with magnetospheric substorms. This, of course, raises the question as to the mechanisms which cause these oscillations, and the relationship between oscillating aurora and the substorm.

Several studies have considered the effects of magnetohydrodynamic (MHD) waves on particle precipitation in the ionosphere (Berger, 1963; Davidson, 1990; Milan et al., 2001), and a strong link has been suggested between discrete auroral arcs and MHD waves (Samson et al., 1996). MHD field line resonances (FLRs) are formed through wave coupling of compressional and shear Alfvén waves. Theory describing this coupling is described in Chen and Hasegawa (1974) and Southwood (1974). Theoretical considerations show that the FLR should have a narrow radial localization and a $180^{\circ}$ phase shift of the electric and magnetic wave fields across the center of the resonance, and a similar shift across the belt of auroral oscillation (Walker et al., 1979; Liu et al., 1995). These are testable predictions, and can be compared to photometer observations. The purpose of this letter is to report comparisons of FLR model theoretical predictions with data observations and to report a strong correlation of oscillating aurora with substorms. This gives some insight into the source and modulation mechanism of

Copy right (C) The Society of Geomagnetism and Earth, Planetary and Space Sciences (SGEPSS); The Seismological Society of Japan; The Volcanological Society of Japan; The Geodetic Society of Japan; The Japanese Society for Planetary Sciences. the precipitating protons and electrons that are responsible for the luminosity variations observed by photometers.

In terms of the substorm, it is not clear what type of disturbance excites compressional modes at expansive phase onset. Whatever the disturbance is, it affects the magnetosphere by exerting stresses in the near-earth regions. The local compression of the configuration generates compressional MHD waves that encounter positive gradients in the Alfvén velocity that lead to the excitation a shear Alfvén waves on magnetic shells distant from the source of the onset. The model of Liu et al. (1995) considers the dynamics of the substorm recovery phase, the period immediately following onset, and thus after the excitation of compressional modes which propagate the substorm disturbance through the magnetosphere. We will compare data from photometer observations with model predictions. After substorm onset, energy from the compressional mode can radiate downtail and towards the flanks. This resonant absorption occurs when compressional mode energy is converted in the inner magnetotail and at the plasma sheet boundary layer. As the compressional wave front expands to these locations the Alfvén velocity gradient is such that resonant shear Alfvén waves will be set up. Because of the coupling of energy to spatially different locations, auroral activity is expected to occur in distinct high- and low-latitude locations. Theory predicts that the high-latitude activity will feature a poleward phase shift of $180^{\circ}$ across the belt of auroral oscillation (Liu et al., 1995; Hughes, 1983). Similarly, the low-latitude activity should exhibit a $180^{\circ}$ equatorward phase shift and a temporally varying parallel electric field will be established along the resonant field line (Block and Fälthammer, 1990; Liu et al., 1995). A consequence of this parallel electric field is that precipitation of protons and electrons will be anticorrelated. If there were no parallel electric field there should be no such phase difference and the precipitation of protons 

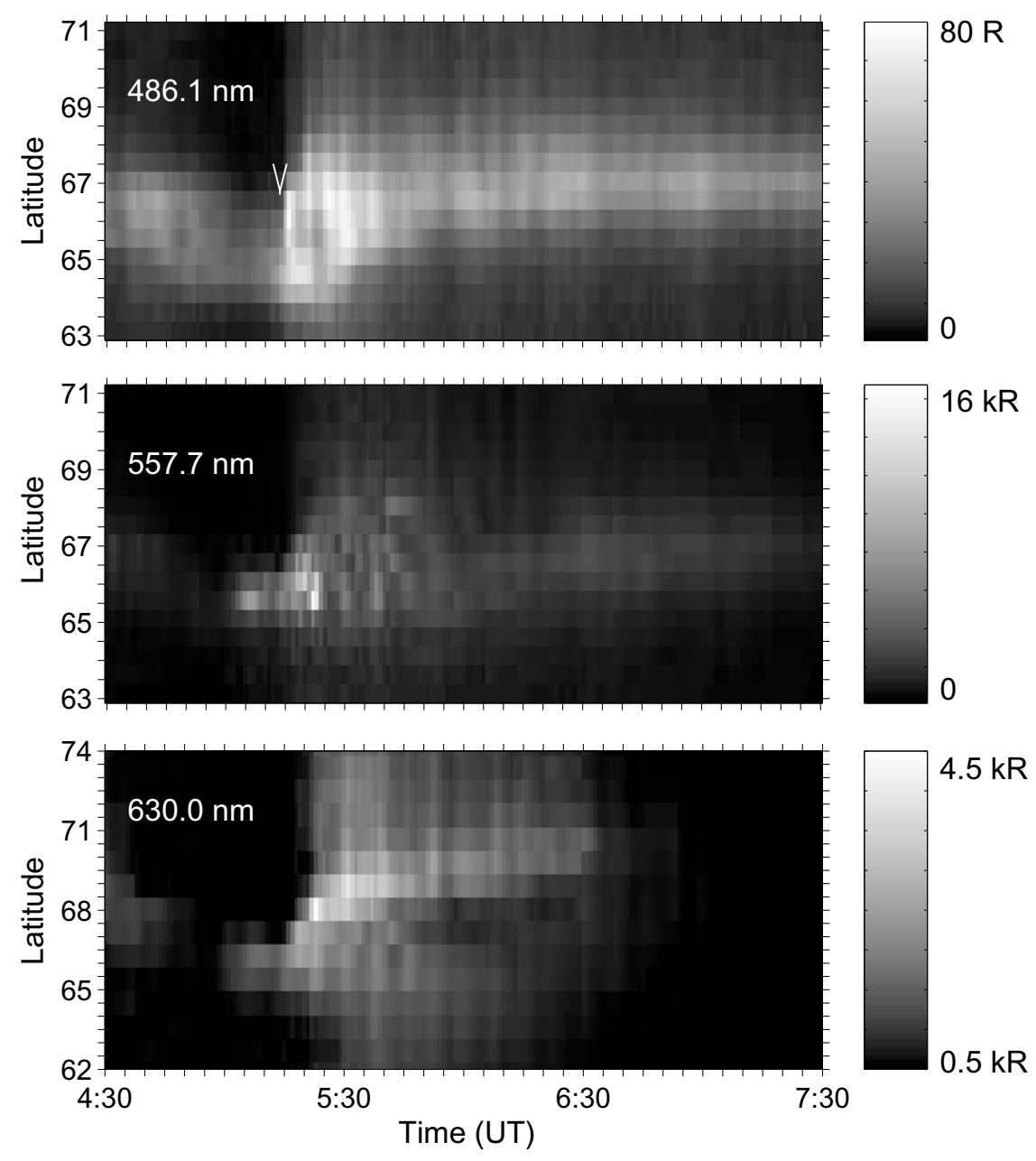

Fig. 1. Keograms of intensity of auroral emissions from the Gillam meridian scanning photometer measured on April 1, 2000. Data are shown for three wavelengths, at 486.1, 557.7, and 630.0-nm. The estimated time of substorm expansive phase onset is indicated by the arrow on the top plot.

and electrons would likely be similarly modulated (Liu et al., 1995).

\section{Data Observations and Analysis}

In this section, we test the theoretical predictions with observations from the Gillam photometer station. The time resolution of the photometer is two complete scans per minute, and scans are performed along a meridian closely aligned with geomagnetic north. A magnetospheric substorm on April 1, 2000 is observed after approximately 0420 UT, with expansive phase onset at about 0525 UT. Prior to onset, growth phase aurorae were observed with the usual equatorward motion, indicating loading and the stretching of magnetotail field lines (Voronkov et al., 1999; Wanliss et al., 2000). Figure 1 shows the photometer keograms from lines sensitive to electron and proton precipitation. The time of onset is indicated by the white line in the uppermost plot. The 486.1-nm $(H \beta)$ emission is caused by precipitation of protons of tens of $\mathrm{keV}, 557.7-\mathrm{nm}$ from hot electrons of several $\mathrm{keV}$, and 630.0-nm from warm electrons of a few hundred $\mathrm{eV}$. Luminosity oscillations are present in each of the three wavelengths shown, but are not clearly visible in the keogram. For $630.0-\mathrm{nm}$, two latitudinal regions of activity can be distinguished, between approximately $66^{\circ}$ to $68^{\circ}$ and $70^{\circ}$ to $72^{\circ}$. Oscillations in the emissions are more easily seen in Fig. 2, which shows a scaled intensity plot of the first differences for the three wavelengths taken at $66.4^{\circ}$. The oscillations for the 557.7-nm and 630.0-nm emissions appear correlated during the entire interval. Between about 05150545 UT there is clear anticorrelation between 486.1-nm and 557.7-nm signals, but any correlation after that is unclear. In Fig. 3 the power spectrogram for this event is shown for the three wavelengths. The spectrogram was obtained by Fourier analysing each latitude-time series and collecting them together in a contour plot as shown. This event is characterised by a resonance structure centered around $2.2 \mathrm{mHz}$, although other peaks are also evident, especially for 630.0-nm near 2.9 and $3.8 \mathrm{mHz}$. The peak around $2.2 \mathrm{mHz}$ has power at lowand high-latitude regions. The higher latitude peak is not clear in $\mathrm{H} \beta$, although its peak in power is spread over nearly 4 degrees in latitude. It is likely that since the proton aurora is more diffuse because of charge exchange experienced by the precipitating protons, it is unable to resolve the poleward most oscillating structure. Figure 4 shows magnetic Xcomponent traces (top) from a latitudinally separated line of magnetometers in the CANOPUS array. Clear pulsations are visible in FCHU and GILL magnetograms. The lower plot shows the power spectrum for the data between 0520-0620 UT from the FCHU station. This indicates that the pulsations are centered somewhere between the GILL and FCHU sta- 


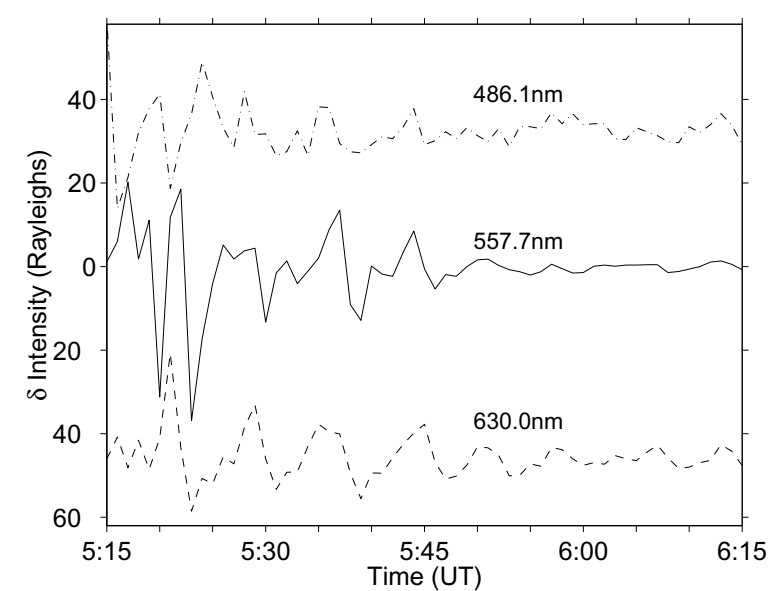

Fig. 2. First differences of the 486.1, 557.7, and 630.0-nm emissions between $0520-0720 \mathrm{UT}$ at $66.4^{\circ}$. The 557.7 and $630.0-\mathrm{nm}$ emissions have been scaled by factors of 150 and 20 , respectively, for easier comparison.
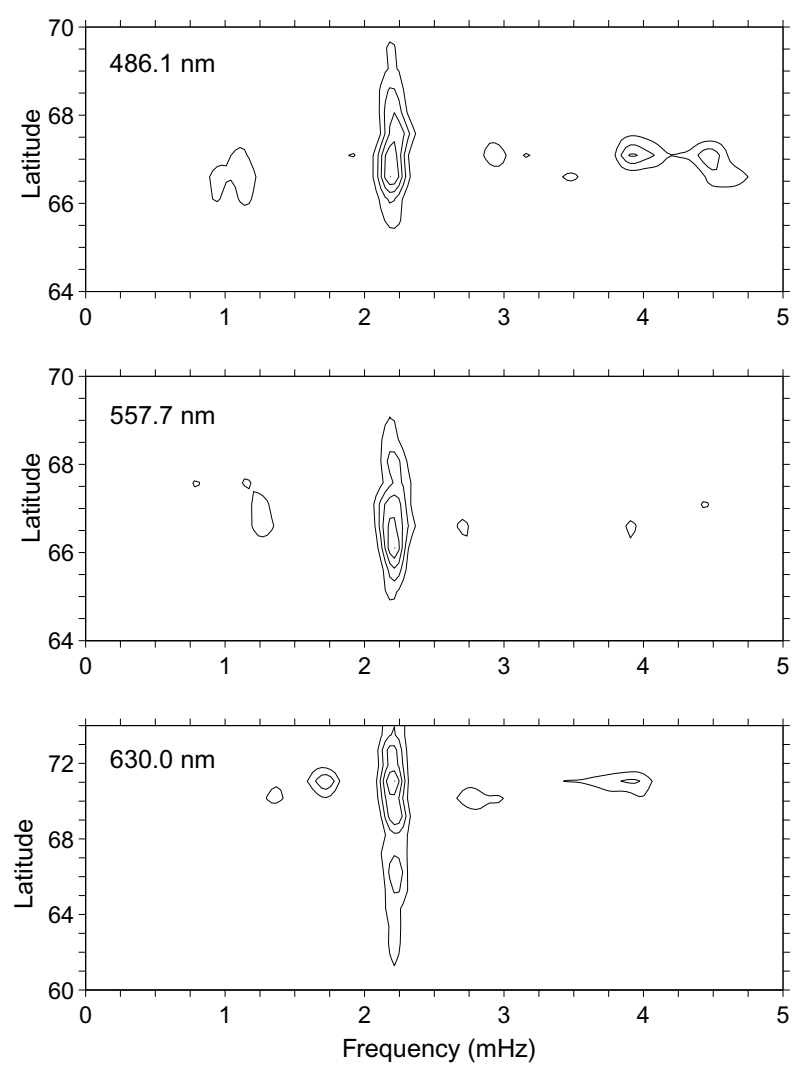

Fig. 3. Contour plots of power spectra for 486.1, 557.7, and 630.0-nm. Power spectra were computed from the time series of each latitudinal bin.

tions (67.4 and 69.7 degrees geomagnetic latitude), and has a frequency about $2.2 \mathrm{mHz}$.

A test of the phase correlation between protons and electrons can be considered via the following measure applied to the optical emissions

$$
\sigma=\frac{\int \delta I_{486.1}(t) \delta I_{557.7}(t)}{\int\left|\delta I_{486.1}(t)\right|\left|\delta I_{557.7}(t)\right|}
$$

where $\delta I$ is the signal with the static part removed. The
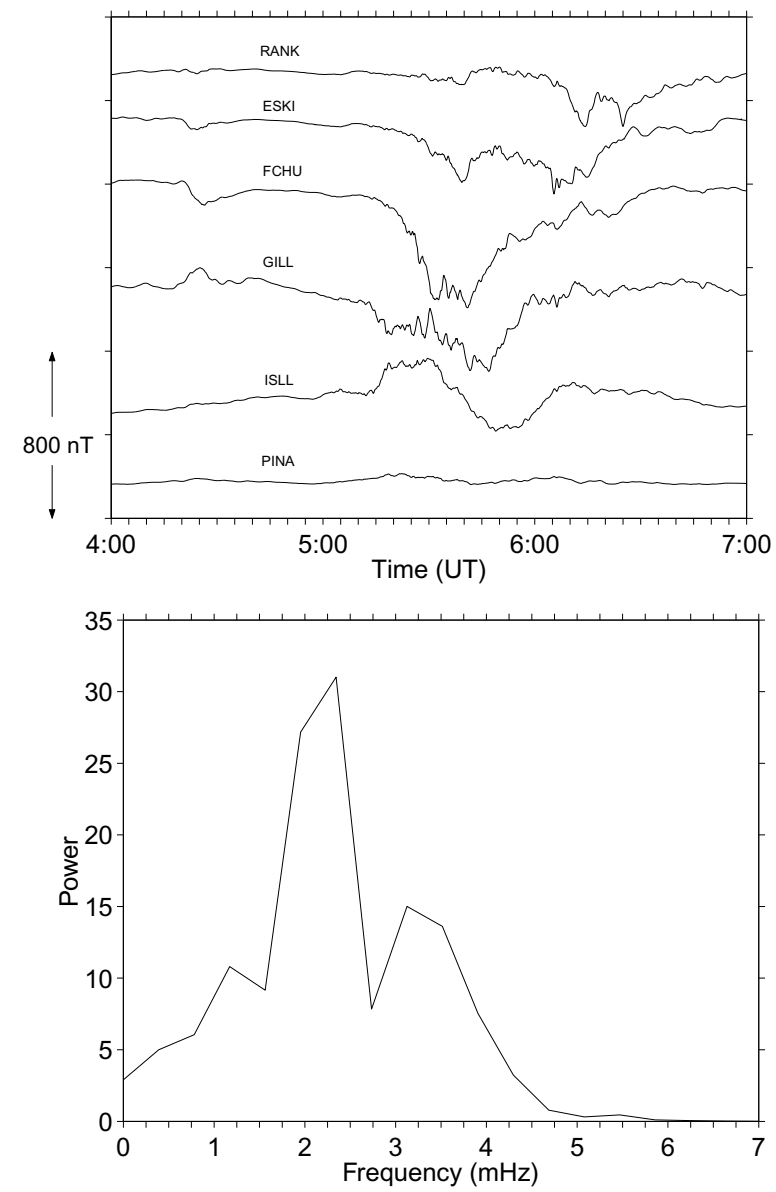

Fig. 4. (top) Magnetic X-component traces from a latitudinally separated line of magnetometers in the CANOPUS array. Clear pulsations are visible in FCHU and GILL magnetograms (see Rostoker et al., 1995 for the station locations). (bottom) Power specrum for the data between 0520-0620 UT from the FCHU station.

correlation was computed between 0520-0620 UT and we found that at latitudes of $66.6^{\circ}, 66.1^{\circ}$, and $65.6^{\circ} \sigma=-0.55$, $-0.84,-0.33$, respectively. Whereas a correlation of $\sigma=$ -1 implies the signals are out of phase, +1 means the signals are perfectly in phase, and a value near 0 means the time series are either uncorrelated or out of phase. Our results indicate an anticorrelation, and thus are consistent with a FLR mechanism and a parallel electric field. This is different from the result of Liu et al. (1995) who found a positive correlation for the event they considered, although this may be due to the limited spatial resolution of the Gillam photometer. This difference reflects the possibility that the precipitation process may involve competing mechanisms which affect the overall signature observed by photometers. A further quantitative test is to see whether the latitudinal phase shifts across the auroral optical resonances show the predicted phase pattern. The upper plot in Fig. 5 shows the power and phase shift across the $2.2 \mathrm{mHz}$ resonance for the 557.7-nm emissions. The phase shift across the peak at $\sim 66^{\circ}$ is about $120^{\circ}$ running from large to small values. This is the expected theoretical pattern for the lower latitude resonance, although the phase shift should be a full $180^{\circ}$. No clear power peak is observed at higher latitudes, but there is a $180^{\circ}$ phase shift running from low to high values across about $68.5^{\circ}$, suggesting 

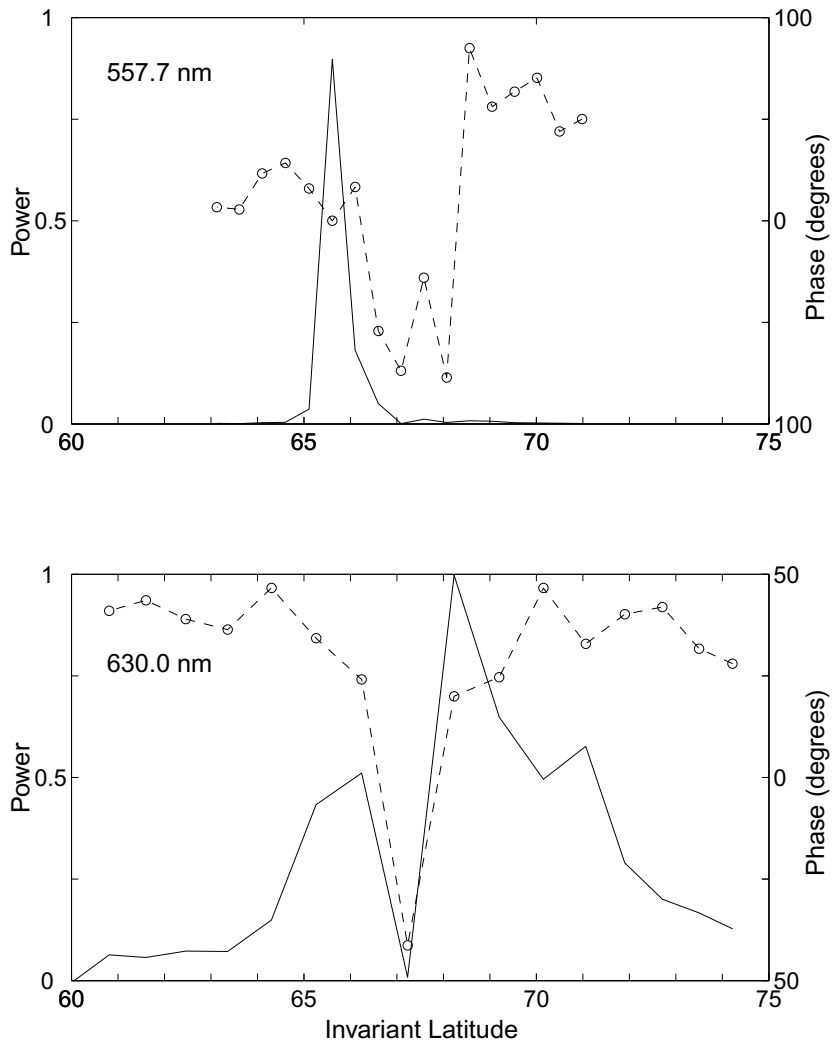

Fig. 5. Latitudinal phase (dashed) and power (solid) distributions of the 2.2 $\mathrm{mHz}$ power for 557.7 and 630.0 -nm emissions.

the presence of a higher latitude resonance. The lower figure shows the phase change across the resonance for 630.0-nm. Two clear peaks in power are at $\sim 66^{\circ}$ and $\sim 69^{\circ}$. Whereas the phase change across the lower latitude peak is from $50^{\circ}$ to $-50^{\circ}$, it is from $-50^{\circ}$ to $50^{\circ}$ for the higher latitude peak. Thus the phase change across the low- and high-latitude resonances are qualitatively correct, although not the full $180^{\circ}$ expected from theory.

\section{Discussion}

In our investigation of photometer data from the Gillam station of the CANOPUS array we found luminosity oscillations frequently associated with magnetospheric substorms. Detailed statistics of this relationship will be reported later. The primary purpose of this letter has been to present evidence supporting the view that modulation of the substorm associated auroral particle precipitation pattern is due to resonant Alfvén waves which carry parallel electric field. The association of parallel electric fields with Alfvén waves has been noted previously (Hasegawa, 1976; Goertz and Boswell, 1979; Rankin et al., 1997; Keiling et al., 2000). The data presented here, for the substorm recovery phase on April 1, 2000, support the concept that compressional waves generated during expansive phase onset are resonantly converted at inner and outer boundaries of the plasma sheet to shear Alfvén waves which carry parallel electric field.

This event is helpful for comparison with theory since luminosity oscillations exist in two distinct latitudinal bands and frequencies around $2.2 \mathrm{mHz}$. In terms of the MHD theory of the propagating substorm disturbance, the lower- latitude band represents the inner resonance, and the higherlatitude band represents the outer resonance generated after expansive phase onset. These resonances develop after compressional modes couple to shear modes in the inner magnetotail and near the plasma sheet boundary layer. Additional comparisons with theory show agreement, in that the proton and electron auroras over the resonance location are approximately out of phase. Such a phase difference is expected when a parallel electric field develops since the electric field will periodically retard proton precipitation and accelerate electrons. We regard the strongest evidence supporting the mechanism discussed above to be the latitudinal phase shifts across the inner and outer resonances, which have the sense expected by theory (Fig. 5). The phase shifts are not, however, a full $180^{\circ}$, as predicted. This may be because while resonances can be very narrow (a few ion gyroradii), the photometer resolution in our study is over $100 \mathrm{~km}$ and the true latitudinal phase variation may be modified.

Acknowledgments. J. A. W. is grateful to J. C. Samson for encouragment and useful discussions. CANOPUS is owned and operated by the Canadian Space Agency.

\section{References}

Berger, S., Giant pulsations in the magnetic field and pulsating aurora, Planet. Space Sci., 11, 867, 1963.

Block, L. P. and C.-G. Fälthammer, The role of magnetic field-aligned electric fields in auroral acceleration, J. Geophys. Res., 95, 5877, 1990.

Chen, L. and A. Hasegawa, A theory of long-period magnetic pulsations, 1, Steady excitation of field line resonances, J. Geophys. Res., 79, 1024, 1974.

Davidson, G. T., Pitch-angle diffusion and the origin of temporal and spatial structures in morningside aurorae, Space Sci. Rev., 53, 45, 1990.

Fenrich, F. R., et al., ULF high and low-m field line resonances observed with SuperDARN, J. Geophys. Res., 100, 21535, 1995.

Goertz, C. K. and R. W. Boswell, Magnetosphere-ionosphere coupling, J. Geophys. Res., 84, 7239, 1979.

Hasegawa, A., Particle acceleration by MHD surface wave and formation of aurora, J. Geophys. Res., 81, 5083, 1976.

Hughes, W. J., Hydromagnetic waves in the magnetosphere, in SolarTerrestrial Physics: Principles and Foundations, edited by R. L. Carovillano and J. M. Forbes, D. Reidel, Norwell, Mass., 1983.

Jacobs, J. A., et al., Classification of geomagnetic micropulsations, J. Geophys. Res., 69, 180, 1964.

Keiling, A., et al., Large Alfvén wave power in the plasma sheet boundary layer during the expansion phase of substorms, Geophys. Res. Lett., 27, 3169,2000 .

Liu, W. W., et al., Theory and observation of auroral substorms: a magnetohydrodynamic approach, J. Geophys. Res., 100, 79, 1995.

Lyatsky, W., et al., Field line resonance interference model for multiple auroral arc generation, J. Geophys. Res., 104, 263, 1999.

McDiarmid, D. R., et al., Simultaneous observation of a traveling vortex structure in the morning sector and a field line resonance in the postnoon sector, J. Geophys. Res., 99, 8891, 1994.

Milan, S. E., et al., Auroral forms and the field-aligned current structure associated with field line resonances, J. Geophys. Res., 106, 25825, 2001.

Rankin, R., et al., Shear flow vortices in magnetospheric plasmas, Phys. Plasmas, 4, 829, 1997.

Rostoker, G., et al., CANOPUS - a ground-based instrument array for remote sensing the high latitude ionosphere during the ISTP/GGS program, Space Sci. Rev., 71, 743-760, 1995.

Samson, J. C., et al., Substorm intensifications and field line resonances in the nightside magnetosphere, J. Geophys. Res., 97, 8495, 1992.

Samson, J. C., et al., Observations of field line resonances, auroral arcs, and auroral vortex structure, J. Geophys. Res., 101, 17373-17383, 1996.

Southwood, D. J., Some features of field line resonances in the magnetosphere, Planet. Space Sci., 22, 483, 1974.

Voronkov, I., et al., Dynamics of the substorm growth phase as observed using CANOPUS and SuperDARN instruments, J. Geophys. Res., 104, 28491-28505, 1999. 
Walker, A. D. M., et al., STARE auroral radar observations of Pc 5 geomagnetic pulsations, J. Geophys. Res., 84, 3373, 1979.

Wanliss, J. A., et al., On the use of photometer data to map dynamics of the magnetotail current sheet during substorm growth phase, J. Geophys. Res., 105, 27673-27684, 2000

Wanliss, J. A., et al., Field line resonances in a stretched magnetotail:
CANOPUS optical and magnetometer observations, J. Geophys. Res., 2002 (in press).

J. A. Wanliss (e-mail: james.wanliss@erau.edu) and R. Rankin 